\title{
Magnetic cooling for microkelvin nanoelectronics on a cryofree platform
}

\author{
M. Palma, ${ }^{1,}$ a) D. Maradan, ${ }^{1,2, a)}$ L. Casparis, ${ }^{1,3}$ T.-M. Liu, ${ }^{1,4}$ F. Froning, ${ }^{1}$ and D. M. Zumbühl ${ }^{1}$, b) \\ 1) Department of Physics, University of Basel, Klingelbergstrasse 82, CH-4056 Basel, \\ Switzerland \\ ${ }^{2)}$ Physikalisch-Technische Bundesanstalt (PTB), Bundesallee 100, 38116 Braunschweig, \\ Germany \\ ${ }^{3)}$ Center for Quantum Devices, Niels Bohr Institute, University of Copenhagen, 2100 Copenhagen, \\ Denmark \\ ${ }^{4)}$ Department of Applied Physics, National Pingtung University, Pingtung City, \\ Taiwan
}

(Dated: 23 March 2018)

We present a parallel network of 16 demagnetization refrigerators mounted on a cryofree dilution refrigerator aimed to cool nanoelectronic devices to sub-millikelvin temperatures. To measure the refrigerator temperature, the thermal motion of electrons in a Ag wire thermalized by a spot-weld to one of the $\mathrm{Cu}$ nuclear refrigerators - is inductively picked-up by a superconducting gradiometer and amplified by a SQUID mounted at $4 \mathrm{~K}$. The noise thermometer as well as other thermometers are used to characterize the performance of the system, finding magnetic field independent heat-leaks of a few $\mathrm{nW} / \mathrm{mol}$, cold times of several days below $1 \mathrm{mK}$, and a lowest temperature of $150 \mu \mathrm{K}$ of one of the nuclear stages in a final field of $80 \mathrm{mT}$, close to the intrinsic SQUID noise of about $100 \mu \mathrm{K}$. A simple thermal model of the system capturing the nuclear refrigerator, heat leaks, as well as thermal and Korringa links describes the main features very well, including rather high refrigerator efficiencies typically above $80 \%$.

\section{INTRODUCTION}

As thermal excitations represent an ubiquitous energy scale in solid state systems, advancing to lower temperatures might open up the way to the discovery of new physical phenomena such as fragile fractional quantum Hall states 1 and electron-mediated nuclear phase transitions, both in $2 \mathrm{D}$ and $1 \mathrm{D}$ systems 4 . To investigate such phenomena, one needs to access lower temperatures beyond what a dilution refrigerator could achieve. Adiabatic Nuclear Demagnetization (AND) $)^{\sqrt{5 \mid 6}}$ is a well very well established technique with the potential to open the door to the $\mu \mathrm{K}$-regime for nanoelectronics. In many laboratories, the sample is only weakly coupled to the coldest spot of the refrigerator, resulting in sample temperatures significantly higher than the base temperature of the refrigerator. In order to efficiently couple sample and refrigerator, a parallel network of Nuclear Refrigerators (NRs) was proposed $\frac{677}{\text {, }}$, where every lead is well thermalized through the mixing chamber (MC) and has its own NR. Our approach relies on the Wiedemann-Franz cooling of the conduction electrons 8 , which is the main cooling mechanism in the mK-regime and below.

The implementation of a parallel network of NRs on a cryogen-free system is very challenging due to the increased vibration level compared to a wet system. However, cryogen-free platforms will become more important for low temperature experiments, because they offer ample experimental space and operation without liquid helium, thus reducing costs and dependence on helium infrastructures. In addition, particularly referring to AND, cryogen-free systems are suitable for longer precooling

a) These authors contributed equally to this work.

b) dominik.zumbuhl@unibas.ch and extended hold time compared to the traditional wet system, due to liquid He transfers increasing the temperature of the entire system. First operative AND systems on cryogen-free platforms have been implemented using both $\mathrm{PrNi}_{5}$ and $\mathrm{Cu}$ as nuclear refrigerant ${ }^{9}$ 11. In contrast to the single nuclear stage experiment, the parallel network of NRs amends itself for nanoelectronics providing direct cooling of the electrons in each of the wires connected to the sample.

In this article, we present a successful implementation of a parallel network of $\mathrm{Cu}$ NRs on a cryogen-free platform demonstrating cooling with high efficiency close to ideal adiabatic behavior down to $150 \mu \mathrm{K}$. The temperature is measured using an inductive Johnson noise thermometer 12 14, which operates over a broad range of temperatures from $4 \mathrm{~K}$ down to $150 \mu \mathrm{K}$. The noise thermometer is an ideal choice for low temperature applications, because self-heating is reduced due to the inductive readout and the thermometer has the potential to reach the low $\mu \mathrm{K}$-regime. We measure field independent heat leaks of less than $2 \mathrm{nW} / \mathrm{mol}$ for magnetic fields below $1 \mathrm{~T}$, allowing the NRs to stay below $1 \mathrm{mK}$ for roughly 50 hours at $80 \mathrm{mT}$. We model the AND process and obtain a dynamic heat leak independent of the magnetic field ramp rate. Thus, it is possible to increase the efficiency of the AND process by reducing the duration of the $B$-field ramping.

\section{NUCLEAR REFRIGERATOR NETWORK ON A CRYOGEN-FREE PLATFORM}

Recently, AND experiments have been successfully implemented on a cryogen-free platform, using $\mathrm{PrNi}_{5}$ and $\mathrm{Cu}$ as nuclear refrigerant 11 . With $\mathrm{PrNi}_{5}$, reaching ultra-low temperatures is restricted to the rather high 


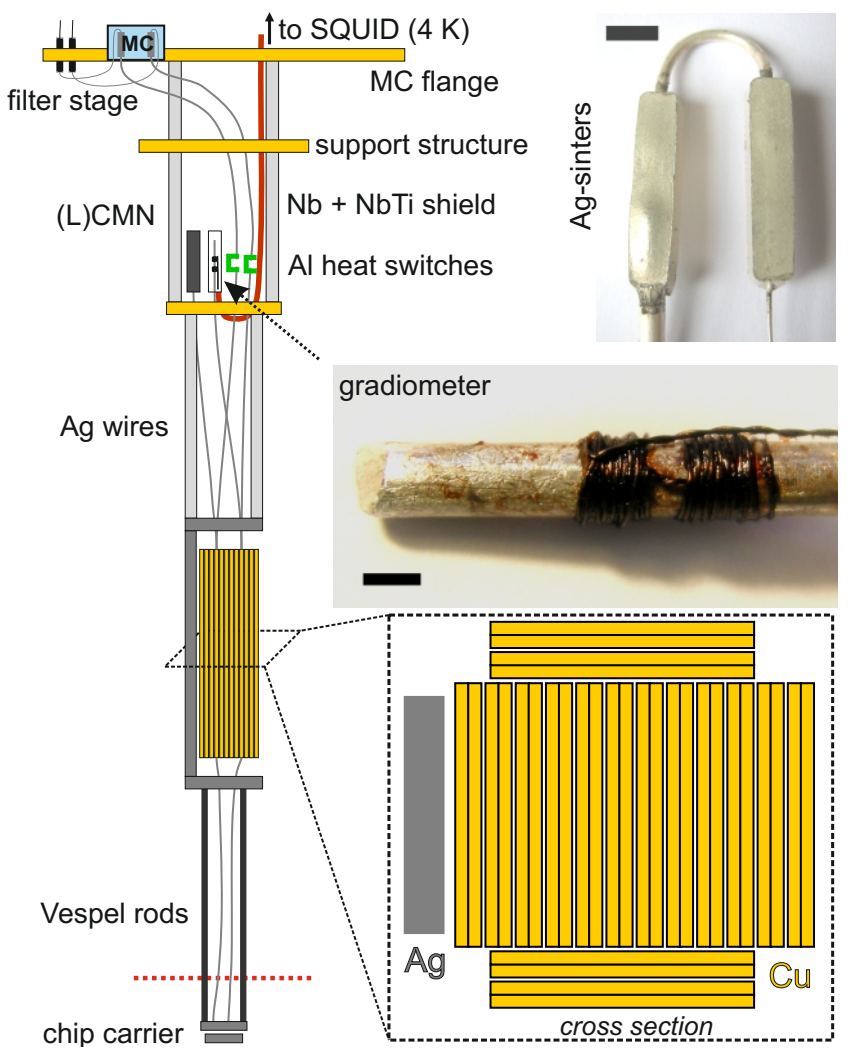

FIG. 1. Schematic of the nuclear demagnetization stage. The measurement leads are thermalized with $\mathrm{Ag}$ powder sinters (top right picture, scale bar: $5 \mathrm{~mm}$ ) in the mixing chamber (MC, blue) and pass through $\mathrm{C}$-shaped $\mathrm{Al}$ heat switches (green) to the $\mathrm{Cu}$ plates. The gradiometer of the noise thermometer as well as the (L)CMN thermometers are positioned in a region of cancelled magnetic field between the $\mathrm{MC}$ and the NR stage. The gradiometer is double-shielded by a $\mathrm{Nb}$ tube and a outer NbTi tube (red). Middle right inset: photograph of the gradiometer pick-up coil made from insulated $\mathrm{Nb}$ wire with $100 \mu \mathrm{m}$ diameter. The $2 \times 20$ turns are wound non-inductively on a high-purity silver wire which is spot-welded to a NR. Scale bar: $2 \mathrm{~mm}$. Lower inset: schematic cross section through the network of 16 parallel NRs.

nuclear ordering temperature $(T \sim 0.5 \mathrm{mK})^{\sqrt{15 \mid 16}}$. In contrast, $\mathrm{Cu}$ can be demagnetized down to the low $\mu \mathrm{K}$ regime and it is very easy to work with and to machine, particularly compared to $\mathrm{PrNi}_{5}$. The high electrical conductivity of $\mathrm{Cu}$ makes it susceptible to eddy current heating, which arises from both ramping of the magnetic field and vibrations in a non-homogenous $B$-field. The pulse tube (PT) cold head is a powerful source of both cooling and vibrations, making the implementation of AND an exacting task. Adiabatic nuclear demagnetization experiments are very susceptible to heat leaks, increasing temperature and accelerating the warm up of the NRs, thus reducing the hold time. The concept of a parallel network of $16 \mathrm{Cu}$ NRs presented here overcomes these challenges and leads to a straightforward integration of the AND technique into transport measurement setups.

Figure 1 shows a schematic of the nuclear stage. Starting from the top, the measurement leads are filtered by lossy thermocoax ${ }^{17}$ from room temperature (RT) to the MC flange of the dilution refrigerator. Additional fil- tering is achieved by home-built Ag-epoxy filters $\frac{\sqrt{18}}{}$ and double-stage RC filters bolted to the MC flange. Next, each of the 16 leads is thermalized inside the $\mathrm{Cu} \mathrm{MC}$ using Ag powder sinters, as shown in the top right inset of Fig. 1, which are electrically insulated from the MC (ground) and each other. To allow the passage of the leads through the $\mathrm{MC}$ we designed super-fluid leaktight feedthroughs on the bottom of the MC. The leads exit the $\mathrm{MC}$ as annealed $\mathrm{Ag}$-wires, then pass through the $\mathrm{Al}$ heat switches with fused joints 19 and finally they are spot-welded to $\mathrm{Cu}$ plates. At the bottom of each NR, another annealed $\mathrm{Ag}$ wire continues to the chip carrier, providing a platform for nanostructured samples on an easily exchangeable chip carrier, see Fig. 1. Therefore, each lead provides a thermally highly conductive path from the sample to the NR, electrically insulated from all other wires and ground. The chip socket below the red dashed line in Fig. 1 was not mounted during the measurements in the main text, but can easily be added without significant influence on refrigerator performace. For additional details about the measurement setup see the supplementary materials 20 .

Magnetic fields up to $9 \mathrm{~T}$ can be separately and independently applied to the AND stage and the sample. The $\mathrm{C}$-shaped $\mathrm{Al}$ pieces are used to implement the concept of heat switches allowing to choose between excellent or very poor thermal conductivity, while always keeping the sample electrically connected. In the superconducting state $\mathrm{Al}$ is a thermal insulator while in its normal state, when the superconductivity is broken by a small magnetic field $(\geq 10 \mathrm{mT})$, it is an excellent thermal conductor. All the thermometers used in the experiment are susceptible to magnetic fields; therefore they are positioned together with the $\mathrm{Al}$ heat switches in a region of canceled magnetic field between the MC and the NRs and are double shielded by $\mathrm{Nb}$ and NbTi tubes. The three thermometers in use are a Cerium Magnesium Nitrate (CMN) thermometer, a Lanthanum diluted CMN (LCMN) thermometer and the Johnson noise thermometer. Each thermometer is connected to its own NR through a massive $\mathrm{Ag}$ wire of $25 \mathrm{~cm}$ length.

Although there are no mechanically moving parts in state-of-the-art pulse tubes, vibrations caused by highpressure gas oscillations and the compressor package are transduced to the refrigerator. Despite significant progress in recent years, cryogen-free systems tend to have drastically increased vibration levels compared to standard systems (i.e. dewars with cryo liquids). To account for these challenges special care was taken on damping all connections to the fridge and decoupling the PT cold head ${ }^{21}$ from the rest of the system. The presented setup was improved from a previous wet system 6 (6/7 to particularly meet the demands of a cryogen-free system 21. We introduced a rigid support structure and an adapted geometry of the NRs. Compared to the wet system version ${ }^{8}$, we decreased the cross section relevant for eddy current heating and simultaneously doubled the amount of $\mathrm{Cu}$ per plate. Further, the surface area of the Ag-sinters was tripled to now $9 \mathrm{~m}^{2}$ per lead and the diameter of the $\mathrm{Ag}$ wires is doubled, since these thermal resistances have been identified as a bottle neck during 


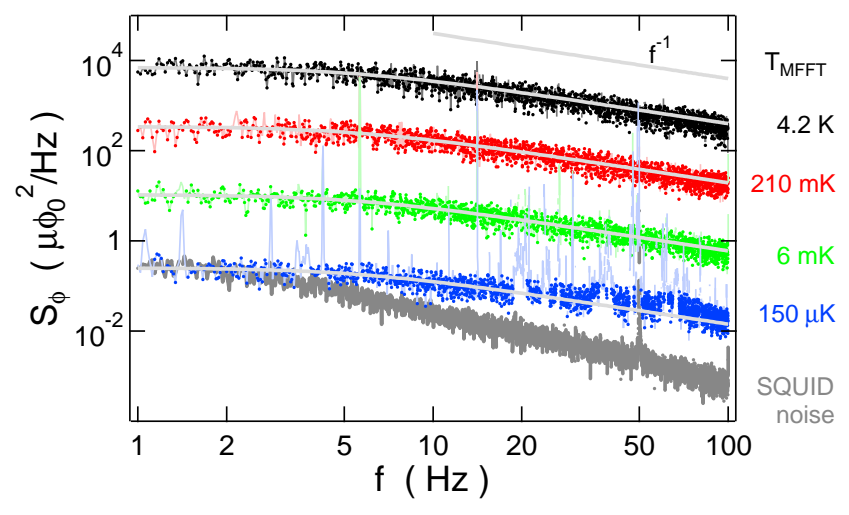

FIG. 2. Power spectral density $S_{\phi}\left(0, T_{\mathrm{MFFT}}\right)$ of the magnetic flux noise, in units of the flux quantum $\phi_{0}$, at various NR temperatures. The light gray solid curves are fits using Eq. (2) which are converted to $T_{\mathrm{MFFT}}$ as described in the text using the reference spectrum at $T_{\text {ref }}=4.2 \mathrm{~K}$. The noise peaks become more visible at lower temperatures where the thermal noise becomes smaller. The SQUID noise shown here in grey is from a similar SQUID with the inputs shorted, not from the SQUID used to measure the MFFT.

precooling 6 .

\section{NOISE THERMOMETRY}

Measuring temperatures in the $\mu \mathrm{K}$ regime is a challenging task, since many thermometers suffer from selfheating and are very susceptible to heat leaks, often leading to a saturation of the thermometer. Here we use a specific type of noise thermometer, the magnetic field fluctuation thermometer (MFFT), based on an inductive read-out 12 14/22 using a SQUID amplifier, which is designed to reduce internal and external heat leaks. In fact, we reduce the external heat leaks onto the thermometer by mechanical and electrical decoupling of shield and thermal noise source, see supplementary material. In addition, thanks to the inductive read-out, the thermal noise is detected without any bias applied, thus reducing self-heating of the thermometer. Our improvements allow us to measure temperatures down to $150 \mu \mathrm{K}$, whereas the lowest measurable temperature is roughly $100 \mu \mathrm{K}$ given by the SQUID noise floor. Figure 2 shows the spectra for various temperatures. Note that for the lowest temperature (blue) the spectrum is just above the SQUID noise spectrum (grey).

The temperature of electrons in a metal is related to their thermal (Brownian) motion, which generates current noise given by the Johnson-Nyquist formula ${ }^{23 \mid 24}$ :

$$
S_{I}=\frac{4 k_{B} T}{R} .
$$

Here, $S_{I}$ is the power spectral density of the current noise, $k_{B}$ is the Boltzmann constant, $T$ is the temperature of the electrons in the conductor and $R$ is the resistance of the metal. The read-out of the thermal noise is done by a gradiometer, consisting of two counterwound superconducting pick-up coils (detector) wrapped tightly around a Ag wire (thermal noise source) of radius $r$. The working principle is the following: thermal currents are transformed, by self-inductance, into magnetic flux fluctuations, detected by the pick-up coil. In the low frequency range, the power spectral density of the magnetic flux noise $e^{22 \mid 25}$ can be written as $S_{\phi}\left(0, T_{\mathrm{MFFT}}\right)=4 k_{B} T_{\mathrm{MFFT}} \sigma G \mu_{0}^{2} r^{3} / 2 \pi$, where $\mu_{0}$ is the vacuum permeability, $T_{\mathrm{MFFT}}$ is the electronic temperature measured with the MFFT, $G$ is a geometric factor $^{22}$ and $\sigma$ is the electrical conductivity at low temperatures, which is assumed temperature independent in the $\mathrm{mK}$ range. The electrical conductivity is defined as $\sigma=\sigma_{\mathrm{RT}} * R R R$, with RRR being the residual resistivity ratio and $\sigma_{\mathrm{RT}}$ the room temperature conductivity. Note that $S_{\phi}\left(0, T_{\mathrm{MFFT}}\right)$ depends linearly on temperature $T_{\mathrm{MFFT}}$, since $\sigma$ is constant at low frequency where the Skin effect is negligible. Figure 2 shows that all the spectra have a low frequency plateau.

The Skin effect forces high frequency current fluctuating to the metal surface. As a consequence the conductivity of the $\mathrm{Ag}$ wire becomes frequency dependent, resulting in a low-pass like shape of $S_{\phi}\left(f, T_{\mathrm{MFFT}}\right)$. Such a frequency dependence is described by the following equation:

$$
S_{\phi}\left(f, T_{\mathrm{MFFT}}\right)=\frac{S_{\phi}\left(0, T_{\mathrm{MFFT}}\right)}{\sqrt{1+\left(\frac{f}{f_{c}}\right)^{2}}},
$$

where the cut-off frequency is given by $f_{c}=$ $4.5 /\left(\pi \mu_{0} \sigma r^{2}\right)^{22}$. As expected in Fig. 2 the amplitudes of the spectra decrease as $1 / f$ at high frequencies.

In order to measure ${ }^{26}$ the power spectral density, we need to acquire 10 real-time noise traces with $50 \mathrm{~s} \mathrm{du}$ ration each, which are averaged after Fourier transformation. Noise peaks appear mostly at frequencies corresponding to higher harmonics of the rotation frequency of the PT motor $(1.4 \mathrm{~Hz})$, see light blue spectrum in Fig. 2 . The peaks become more evident at low temperatures due to the lower thermal noise background. To eliminate these peaks we fit every spectrum first with a polynomial of $10^{\text {th }}$ order and eliminate any data that exceeds the polynomial by more than an empirically determined threshold factor.

The MFFT is used as a secondary thermometer calibrated against the MC thermometer at $4.2 \mathrm{~K}$. From the fit of Eq. (2) to the reference spectrum at $4.2 \mathrm{~K}$, we extract a value of $f_{c} \sim 5 \mathrm{~Hz}$ independent of temperature and corresponding to a RRR of about 2000, which matches typical RRRs determined in independent transport measurements. Thus, we fix $f_{c}=5 \mathrm{~Hz}$ and extract $T_{\mathrm{MFFT}}$ as the only fit parameter from the fit to Eq. 22).

To achieve optimal performance of the MFFT, we addressed and solved the following technical issues. First, to avoid effects from homogeneous magnetic fields, the pick-up coil is non-inductively wound around the $\mathrm{Ag}$ wire. Second, the gradiometer shown in Fig. 1 is connected by a long section of twisted $\mathrm{Nb}$ wires to the SQUID, which is anchored to the quasi- $4 \mathrm{~K}$-flange of the refrigerator. The twisted $\mathrm{Nb}$ wires are double-shielded with a $\mathrm{Nb}$ and $\mathrm{NbTi}$ tube, both thermalized at the mixing chamber plate, cold 
plate and still plate. Finally, we mounted the SQUID at $4 \mathrm{~K}$ to avoid low-frequency excess flux noise ${ }^{27}$, which can arise at sub-K temperatures.

\section{NUCLEAR REFRIGERATOR PERFORMANCE}

The nuclear refrigerator technique is based on a single shot cycle consisting of the following steps: magnetization in an initial field $B_{\mathrm{i}}=9 \mathrm{~T}$, precooling down to $T_{\mathrm{i}}=10 \mathrm{mK}$ (three days), and demagnetization down to a final field $B_{\mathrm{f}}$. Finally, low temperatures can be explored over a period of time while the system continuously warms up, due to a small parasitic heat leak $(\dot{Q})$ absorbed by the NRs. During the AND process the nuclear temperature of the $\mathrm{Cu}$ plate is lowered from the initial temperature $T_{i}$ down to the final temperature $T_{f}$. During magnetization and precooling, the $\mathrm{Al}$ heat switches are set to conduct heat excellently (normal state) to cool the NRs via the MC. While demagnetizing and warming up, the heat switches are superconducting to prevent heat flowing from the MC into the NRs. In this refrigeration technique, the nuclear spin degree of freedom has by far the largest heat capacity, absorbing the heat leaks coming into the NRs. This can lead to non-equilibrium configurations where other degrees of freedom (e.g. electrons, phonons) can be at different temperatures than the nuclear spins, due to the finite thermal conductivity between them.

We need to characterize the heat leak of the system, which then defines the efficiency $\xi=\left(T_{\mathrm{i}} / T_{\mathrm{f}}\right) /\left(B_{\mathrm{i}} / B_{\mathrm{f}}\right)$ of the AND process. An efficiency of $100 \%$ indicates a fully adiabatic and reversible process while $\xi$ less than $100 \%$ signifies the presence of heat leaks, which spoil the adiabaticity of the AND process. One distinguishes two types of heat leaks: a static heat leak $(\dot{Q})$ appearing already at fixed magnetic field and attributed mainly to heat release, radiation and vibrations. Beyond that, an additional dynamic heat leak $\left(\dot{Q}_{\mathrm{dyn}}\right)$ appears when sweeping the magnetic field.

To determine the static heat leak onto a NR, we read a sensor temperature $T_{\mathrm{s}}$ as a function of time during the warm up, see Fig. 3 , displaying $T_{\mathrm{s}}^{-1}$. Our sensor cannot operate directly on the NR due to the magnetic fields present, and thus is placed at some distance and is thermally well connected to the NR through a highconductivity Ag wire. Over time, the temperature is continuously increasing until it saturates at rather high temperature $\sim 50 \mathrm{mK}$, far exceeding the MC temperature $T_{\mathrm{MC}} \sim 7 \mathrm{mK}$. At this point, the heat leaking from the NR through the $\mathrm{Al}$ heat switches into the MC balances the static heat leak, keeping the $\mathrm{Cu}$ stage at a constant temperature. One can model the warm up behavior of the NRs $s^{5115}$ by assuming a constant static heat leak $\dot{Q}$ flowing entirely into the $\mathrm{Cu}$ nuclear spins:

$$
T_{\mathrm{e}, \mathrm{Cu}}^{-1}(t)=T_{\mathrm{ex}}^{-1}-t\left(\frac{\lambda_{n} B_{f}^{2}}{\mu_{0} \dot{Q}}+\kappa\right)^{-1},
$$

where $T_{\mathrm{e}, \mathrm{Cu}}$ is the electronic temperature of the $\mathrm{Cu}$ plate, $T_{\text {ex }}$ is the extrapolated electronic temperature of the $\mathrm{Cu}$ plate at the beginning of the warm up, $\mu_{0}$ is the vacuum permeability, $\lambda_{n}$ is the molar nuclear Curie constant of $\mathrm{Cu}$ and $\kappa$ is the Korringa constant $\mathrm{t}^{5}$ for $\mathrm{Cu}$. The Korringa constant quantifies thermal coupling and thus the temperature gradient between the electrons and the nuclei. As Eq. 30 shows, $T_{e, C u}^{-1}$ is an affine function of time. In the intermediate temperature regime, but away from saturation, we fit Eq. (3) to the data, shown as dashed lines in Fig. 3(a). The fits are in very good agreement with the data for intermediate temperatures, which indicates that the heat leak is constant over a long period of time.

From the fit we extract $\dot{Q}$ and $T_{\text {ex }}$. The black crosses in the inset of Fig. 3.(b) show the measured $\dot{Q}$ for ANDs at various final fields. As seen, $\dot{Q}$ is roughly $1 \mathrm{nW} / \mathrm{mol}$ and independent of $B_{f}$ below $1 \mathrm{~T}$. This is striking since it indicates negligible eddy current heating. During a warm up, the magnetic field is held constant but eddy current heating could still arise due to vibrations in an inhomogeneous magnetic field: $\dot{Q} \propto(d B / d t)^{2}=$ $[(d B / d \mathbf{r})(d \mathbf{r} / d t)]^{2}$.

As shown in Fig. 3(a), the temperature sensor shows a saturation in the low temperature regime and lies below the theory curve of inverse temperature. Such an elevated sensor temperature $T_{\mathrm{s}}$ can be caused by heat release, e.g. at the thermometer itself. The temperature gradient between the sensor and the NR can be taken into account using a heat flow equation, that fits the sensor temperature of the MFFT in the whole dynamic range. The total static heat leak can be decomposed into a sensor heat leak $\dot{Q}_{\mathrm{s}}$ and a remaining heat leak directly acting onto the NRs. The temperature gradient due to the heat leak $\dot{Q}_{\mathrm{s}}$ can be written as :

$$
T_{\mathrm{s}}^{2}(t)-T_{\mathrm{e}, \mathrm{Cu}}^{2}(t)=\frac{2}{\kappa_{0}} \dot{Q}_{\mathrm{s}} .
$$

The difference between the square of the two temperatures comes from the integration of the thermal conductivity of the metallic link between the sensor and the NR, which is linear in $\mathrm{T}$. The coefficient $\kappa_{0}=\pi^{2} k_{B}^{2} / 3 e^{2} R_{\mathrm{tot}}$, where $e$ is the electron charge, depends on the total resistance $R_{\mathrm{tot}} \sim 1 \mu \Omega$ comprised in similar parts from the spot welded junction between the $\mathrm{Ag}$ wire and the $\mathrm{Cu}$ plate and the resistance of the $\mathrm{Ag}$ wire. Note that the low temperature resistivity is reduced by a $\mathrm{RRR} \sim 2000$, achieved by annealing the high purity $\mathrm{Ag}$ wire. By plugging Eq. (3) into Eq. (4), we obtain $T_{\mathrm{s}}^{-1}$ as function of the time with $\dot{Q}_{\mathrm{s}}$ as an additional fit parameter. The solid blue curves in Fig. 3(a) show the best fit, exhibiting excellent agreement down to the lowest temperatures. The sensor heat leak $\dot{Q}_{\mathrm{s}}$ is between 5 and $20 \%$ of $\dot{Q}$, indicating a rather small heat leak emanating from the MFFT.

Performing a complete AND experiment to extract $\dot{Q}$ for different $B$-fields is very time-consuming. In order to procure $\dot{Q}$ faster, we introduce an abbreviated precool and warm up (PW) cycle: The $\mathrm{Cu}$ stage is precooled at fixed magnetic field and subsequently warms up due to the heat leak $\dot{Q}$, after being thermally isolated from the $\mathrm{MC}$ with the heat switch. Figure 3 (b) shows the warm-up of a PW cycle at a $B$-field of $0.5 \mathrm{~T}$ for all the thermometers in use. For all three sensors, the inverse 


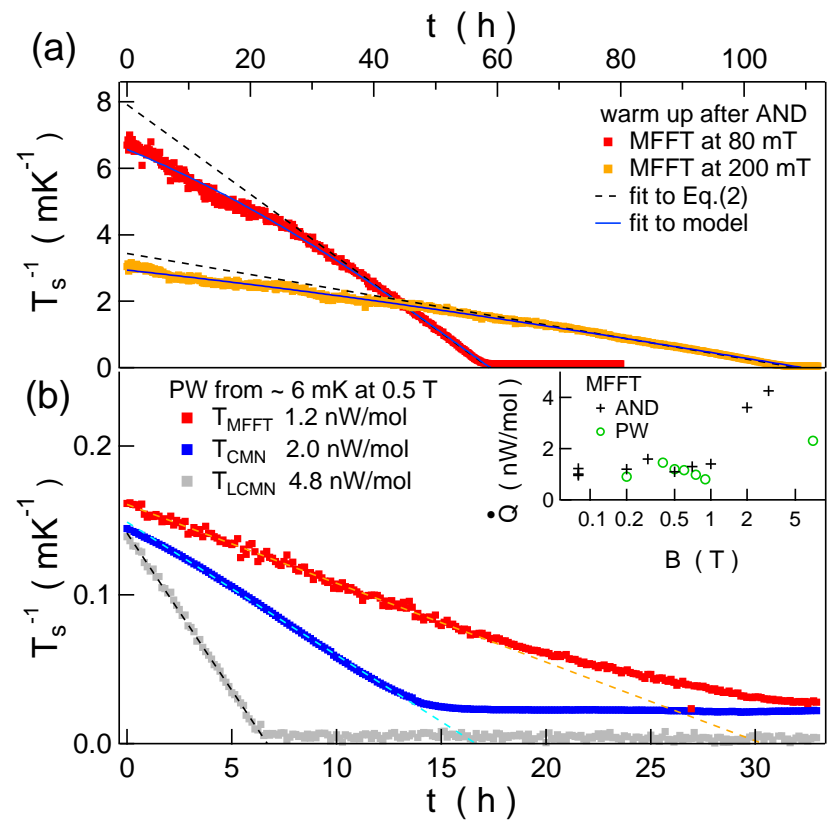

FIG. 3. Warm-up curves: (a) Inverse of $T_{\mathrm{s}}$ measured with the MFFT versus time during the warm up at $80 \mathrm{mT}$ (red squares) and $200 \mathrm{mT}$ (orange squares) after AND. The linear fits (black dashed lines) reveal extrapolated electron temperatures $T_{\mathrm{ex}}=126 / 280 \mu \mathrm{K}$ at the beginning of the warm up and heat leaks of $\dot{Q}=0.9 / 1.16 \mathrm{nW} / \mathrm{mol}$ for $80 \mathrm{mT}$ and $200 \mathrm{mT}$, respectively. The solid blue curves are the fits to the heat flow model (see main text) with $\dot{Q}_{\mathrm{s}}=6 \%$ at $80 \mathrm{mT}$ and $18 \%$ at $200 \mathrm{mT}$ of the total static heat leak. (b) Precool and warm up (PW) measurements: $T_{\mathrm{s}}^{-1}$ during warm up, from MFFT, CMN and LCMN thermometers versus the time after opening the heat switches at $\mathrm{B}=0.5 \mathrm{~T}$, resulting in $\dot{Q}=1.2 / 2.0 / 4.8 \mathrm{nW} / \mathrm{mol}$, respectively. Inset: static heat leak $\dot{Q}$ to the nuclear stage per mol of $\mathrm{Cu}$, measured with the MFFT at various $B$-fields, extracted using Eq. (3) after AND (black crosses) and after PW (green circles).

of the temperature decreases linearly in time and eventually saturates at high temperature. Note that each of the three thermometers have their own saturation temperature (high T) and warm up time, indicating different heat leaks. By using Eq. (3) (dashed line in Fig. 3(b)) we extract a $\dot{Q}$ for the MFFT of around $1 \mathrm{nW} / \mathrm{mol}$ and find higher values of $2 \mathrm{nW} / \mathrm{mol}$ for the $\mathrm{CMN}$ and 4.8 $\mathrm{nW} / \mathrm{mol}$ for the LCMN. The heat leaks extracted with PWs for different $B$-fields are consistent with the ones from warm ups after AND, see inset Fig. 3(b). Note that for the MFFT a minute amount of GE Varnish is used to fix the superconducting pick-up coil to the silver wire while for the packaging of the (L)CMN, a considerable amount of epoxy is used, which is a well-known source of heat release.

Next, we compare the electronic temperature of the $\mathrm{Cu}$ plates $T_{\mathrm{e}, \mathrm{Cu}}$ as extrapolated from the warm up curves after AND $\left(T_{\text {ex }}\right)$ with the measured electronic temperature $T_{\mathrm{MFFT}}$, finding excellent agreement, as seen in Fig. 4 . blue squares. Even though the thermometers used for the extrapolation (CMN and LCMN) become fully saturated at rather high temperatures, here around $2.5 \mathrm{mK}$, the extrapolation method - as also relied on in our previous works $\Phi^{6}-$ is seen here to work rather well down to the lowest temperatures measured. As shown in Fig. 4 below $400 \mu \mathrm{K}, T_{\mathrm{MFFT}}$ starts to be slightly higher than $T_{\mathrm{e}, \mathrm{Cu}}$, reaching a maximum deviation of $20 \%$ for the lowest temperature. At $150 \mu \mathrm{K}$ the MFFT is mainly limited by the SQUID noise level and hence slightly higher than the lowest extracted temperature $T_{\mathrm{ex}}=120 \mu \mathrm{K}$. In the high temperature regime, the MFFT is tested against a calibrated $\mathrm{RuO}_{2}$ thermometer sitting on the MC flange, showing excellent agreement of the temperature reading of the two thermometers, see red squares in Fig. 4

To complete the characterization of the AND system, we now turn to the efficiency of the process. As shown in the inset of Fig. 4 the efficiency decreases monotonically from almost $100 \%$ at high final magnetic field down to $70 \%$ for the lowest final field. The reduction of the efficiency for lower magnetic fields is a result of the smaller heat capacity of the $\mathrm{Cu}$ nuclei, which is proportional to $B_{\mathrm{f}}^{2}$. We simulated the efficiency of the AND process assuming $\dot{Q}_{\text {dyn }}$ depending linearly or quadratic on $B$ or $\dot{B}$ as one would expect for $\dot{Q}_{\mathrm{dyn}}$ arising from vibration or eddy current heating, but in these cases the simulations completely missed the experimental points. In contrast, assuming a fixed $\dot{Q}_{\mathrm{dyn}}$ of $29 \mathrm{nW} / \mathrm{mol}$, independent $B$ or $\dot{B}$, reproduces the data well (green dashed curve in inset of Fig. 4). Thus, the simulation suggests that $\dot{Q}_{\text {dyn }}$ is constant in time and independent of the ramp-rate of the $B$-field, which gives the opportunity to increase the efficiency by reducing the duration of the demagnetization process. This hypothesis was successfully tested in the experiments by doubling the ramp speed of the AND, as shown by the blue crosses in the inset, where $\xi$ increases significantly for the faster rate - albeit the simulation predicts slightly different efficiencies than those measured.

To estimate the dynamic heat leak, we first open the switches, then we ramp the magnetic field from zero to a finite $B$-field and back to avoid any nuclear contribution to the heat capacity. We measured the temperature of the $\mathrm{Cu}$ plates and by integrating the electron heat capacity we obtain the energy stored in the system. In addition, we estimate the heat leaking through the superconducting $\mathrm{Al}$ heat switches due to phonon-dislocation scattering processes 515128 . This estimation yields $\dot{Q}_{\mathrm{dyn}}$ of $22 \mathrm{nW} / \mathrm{mol}$ for a ramp-rate of $1 \mathrm{~T} / \mathrm{h}$, which is comparable to the value used in the simulation. However, the $\dot{Q}_{\text {dyn }}$ estimated with this protocol is dependent on the ramp-rate, which is in disagreement with the simulation. Our simple model needs further work to fully understand the origin of $\dot{Q}_{\mathrm{dyn}}$ and its dependence on the sweep rate.

\section{v. CONCLUSIONS}

In summary, we have implemented a parallel network of 16 electrically separated NRs on a cryogen-free platform. These 16 plates are part of the measurement leads and can be straightforwardly used to cool nanostructured samples. The nuclear stage is equipped with a magnetic field fluctuation thermometer, showing excellent agree- 


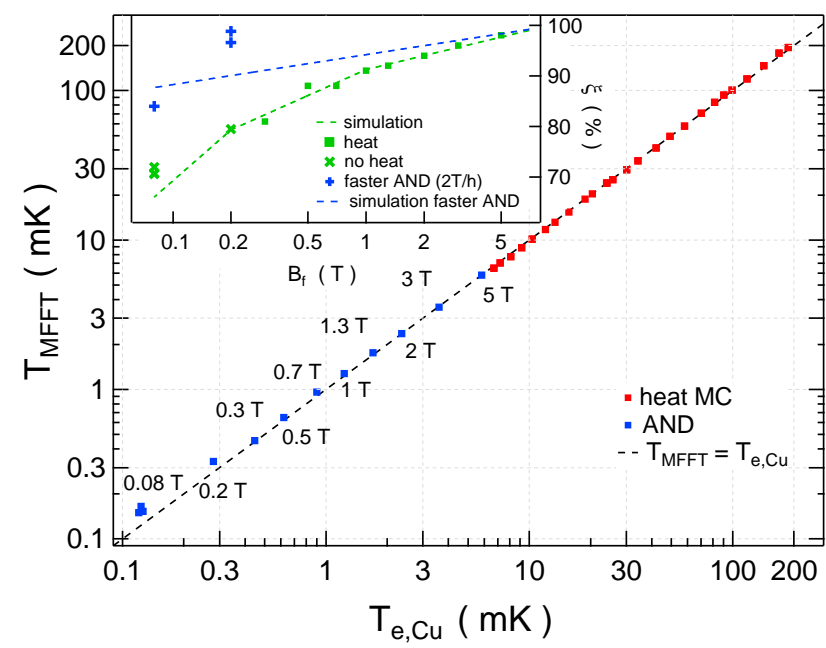

FIG. 4. Temperature measured by the noise thermometer $T_{\text {MFFT }}$ versus temperature of the nuclear stage $T_{\mathrm{e}, \mathrm{Cu}}$. Above $7 \mathrm{mK}, T_{\mathrm{e}, \mathrm{Cu}}$ is measured with a calibrated $\mathrm{RuO}_{2}$ thermometer sitting on the $\mathrm{MC}$ (red squares). In this temperature range the $\mathrm{MC}$ and the $\mathrm{Cu}$ stage are well thermally coupled. For temperatures below $7 \mathrm{mK}, T_{\mathrm{e}, \mathrm{Cu}}$ is extracted from warm ups after AND (blue squares) (see text and Fig. 3(a)). The black dotted line represents $T_{\mathrm{MFFT}}=T_{\mathrm{e}, \mathrm{Cu}}$. Inset: the efficiency $\xi$ as function of the final magnetic fields $B_{\mathrm{f}}$. The dashed curves show simulations of the AND process, carried out at two different ramp speeds. The markers show the efficiency extracted from different AND runs as labeled.

ment with the NR temperature $T_{\mathrm{e}, \mathrm{Cu}}$ down to $400 \mu \mathrm{K}$. After AND to $B_{f}=0.08 \mathrm{~T}$, the lowest temperature reading is limited to $150 \mu \mathrm{K}$ while the extrapolated electron temperatures is $120 \mu \mathrm{K}$, indicating good agreement between the model and measurements. The heat leak measured on the NRs is around $1 \mathrm{nW} / \mathrm{mol}$ and allows the AND stage to stay below $1 \mathrm{mK}$ for roughly 50 hours, see supplementary material20. Higher $B_{f}$ allow for even longer hold times, while still supplying reasonably low temperatures. In addition, we characterized the dynamic heat leak, which appears to be constant in time and independent of the sweep rate of the magnetic field, making possible significantly increased efficiency at faster magnetic field sweep rates.

\section{ACKNOWLEDGMENTS}

We would like to thank H.J. Barthelmess, R. Blaauwgeers, G. Pickett, M. Steinacher and P. Vorselman for useful input and discussions. The work shop team of S. Martin is acknowledged for technical support. This work was supported by the Swiss NSF, NCCR QSIT, the Swiss Nanoscience Institute, the Europeam Microkelvin Platform, an ERC starting grant (DMZ), and EU-FP7
MICROKELVIN and SOLID.

${ }^{1}$ W. Pan, J. S. Xia, V. Shvarts, D. E. Adams, H. L. Stormer, D. C. Tsui, L. N. Pfeiffer, K. W. Baldwin, and K. W. West, Phys. Rev. Lett. 83, 3530 (1999).

${ }^{2}$ P. Simon and D. Loss, Phys. Rev. Lett. 98, 156401 (2007).

${ }^{3}$ P. Simon, B. Braunecker, and D. Loss, Phys. Rev. B 77, 045108 (2008).

${ }^{4}$ C. P. Scheller, T.-M. Liu, G. Barak, A. Yacoby, L. N. Pfeiffer, K. W. West, and D. M. Zumbühl, Phys. Rev. Lett. 112, 066801 (2014).

${ }^{5}$ G. Pickett, Physica B: Condensed Matter 280, 467 (2000).

${ }^{6}$ A. C. Clark, K. K. Schwarzwälder, T. Bandi, D. Maradan, and D. M. Zumbühl, Rev. Sci. Instrum. 81, 103904 (2010).

${ }^{7}$ A. V. Feshchenko, L. Casparis, I. M. Khaymovich, D. Maradan, O.-P. Saira, M. Palma, M. Meschke, J. P. Pekola, and D. M. Zumbühl, Phys. Rev. Applied 4, 034001 (2015)

${ }^{8}$ L. Casparis, M. Meschke, D. Maradan, A. C. Clark, C. P. Scheller, K. K. Schwarzwälder, J. P. Pekola, and D. M. Zumbühl, Rev. Sci. Instrum. 83, 083903 (2012).

${ }^{9}$ G. Batey, A. Casey, M. N. Cuthbert, A. J. Matthews, J. Saunders, and A. Shibahara, New Journal of Physics 15, 113034 (2013).

${ }^{10}$ A. Casey, F. Arnold, L. V. Levitin, C. P. Lusher, J. Nyéki, J. Saunders, A. Shibahara, H. v. d. Vliet, B. Yager, D. Drung, T. Schurig, G. Batey, M. N. Cuthbert, and A. J. Matthews, J. Low Temp. Phys. 175, 764 (2014).

${ }^{11}$ I. Todoshchenko, J.-P. Kaikkonen, R. Blaauwgeers, P. J. Hakonen, and A. Savin, Rev. Sci. Instrum. 85, 085106 (2014).

12 J. Engert, J. Beyer, D. Drung, A. Kirste, and M. Peters, Int. J. Thermophys. 28 (2007).

${ }^{13}$ J. Engert, D. Heyer, J. Beyer, and H. J. Barthelmess, Journal of Physics: Conference Series 400 (2012).

${ }^{14}$ J. Beyer, D. Drung, A. Kirste, J. Engert, A. Netsch, A. Fleischmann, and C. Enss, Applied Superconductivity, IEEE Transactions on 17, 760 (2007).

${ }^{15}$ F. Pobell, Matter and Methods at Low Temperatures (Springer, Berlin, 2007).

${ }^{16}$ R. Mueller, C. Buchal, H. Folle, M. Kubota, and F. Pobell, Cryogenics 20, 395 (1980).

${ }^{17}$ A. B. Zorin, Rev. Sci. Instrum. 66, 4296 (1995).

${ }^{18}$ C. P. Scheller, S. Heizmann, K. Bedner, D. Giss, M. Meschke, D. M. Zumbühl, J. D. Zimmerman, and A. C. Gossard, Appl. Phys. Lett. 104, 211106 (2014).

${ }^{19}$ N. S. Lawson, Cryogenics 22, 121 (1982).

20 See supplementary material for details regarding the setup, precooling and warm up curves for the MFFT, warm up curves for the LCMN and CMN thermometers, comparison between two different gradiometer designs and different sockets.

${ }^{21}$ BlueFors Cryogenics Oy Ltd., Helsinki, Finland.

${ }^{22}$ D. Rothfuss, A. Reiser, A. Fleischmann, and C. Enss, Appl. Phys. Lett. 103, 052605 (2013).

${ }^{23}$ J. B. Johnson, Phys. Rev. 32, 97 (1928).

${ }^{24}$ H. Nyquist, Phys. Rev. 32, 110 (1928)

${ }^{25}$ T. Varpula and T. Poutanen, J. Appl. Phys. 55, 4015 (1984).

${ }^{26}$ The DC-SQUID is operated in a flux-locked loop mode with XXF-1 electronics $\$ 29$, including a second order Bessel-type lowpass filter $\left(f_{3 \mathrm{~dB}}=10 \mathrm{kHz}\right)$. After a room temperature voltage preamplifier with another low-pass filter $\left(f_{3 \mathrm{~dB}}=1 \mathrm{kHz}\right)$, the signal is acquired with a digital-to-analog converter.

${ }^{27}$ F. C. Wellstood, C. Urbina, and J. Clarke, Appl. Phys. Lett. 50, 772 (1987).

${ }^{28}$ K. Gloos, C. Mitschka, F. Pobell, and P. Smeibidl, Cryogenics 30, 14 (1990).

${ }^{29}$ Magnicon GmbH, Hamburg, Germany. 